\title{
AUTOMATIC DETECTION AND RECOGNITION OF ROAD INTERSECTIONS FOR ROAD EXTRACTION FROM IMAGERY
}

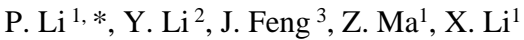 \\ ${ }^{1}$ Chongqing Geomatics and Remote Sensing Center, 404100 Chongqing, China - lpl@d1023.net, 313782848@qq.com, \\ 1x1@d1023.net \\ ${ }^{2}$ School of Civil Engineering and Architecture, Nanchang University, Nanchang 330031, China - 443075372@qq.com \\ ${ }^{3}$ School of Remote Sensing and Information Engineering, Wuhan University, Wuhan,430079, China - fengjt@whu.edu.cn
}

KEY WORDS: Road Extraction, Road Intersection, Segmentation, Shape Analysis, Recognition

\begin{abstract}
:
Automatic road extraction from remote sensing imagery is very useful for many applications involved with geographic information. For road extraction of urban areas, road intersections offer stable and reliable information for extraction of road network, with higher completeness and accuracy. In this paper, a segmentation-shape analysis based method is proposed to detect road intersections and their branch directions from an image. In the region of interest, it uses the contour shape of the segmented-intersection area to form a feature vector representing its geometric information. The extracted feature vector is then matched with some template vectors in order to find the best matched intersection pattern, obtain the type of intersection and the direction of connected roads. The experimental analysis are carried out with ISPRS Vaihingen and Toronto images. The experimental results show that the proposed method can extract most of the road intersections correctly. For the Vaihingen image, the the completeness and correctness are $81 \%$ and $87 \%$, respectfully, while for the Toronto image, the the completeness and correctness are $78 \%$ and $85 \%$, respectfully. It can help to build more correct and complete road network.
\end{abstract}

\section{INTRODUCTION}

With the rapid development of cities, the rapid development of information industry technology makes human activities more and more dependent on geographic information. Accurate and efficient access to geographic information has become a hot area of multidisciplinary research. Traditional spatial data collection and processing methods are difficult to meet the development needs of the industry, and photogrammetry and computer vision provide a technical basis for large-scale and rapid extraction of geospatial data. Automatic and semiautomatic extraction of information of interest from remote sensing data is a requirement of the application and a goal pursued by the photogrammetry and remote sensing industry.

Roads are one of the most important types of geographic information data. The development of society and many industries urgently needs road extraction information in urban scenes. However, affected by the diversity of its own characteristics and the complexity of the surrounding scenes, road extraction has always been a very challenging task. Automatic or semi-automatic road extraction from remote sensing images has been developed for decades. Many new strategies and methods are constantly being used for this, from simple environments in rural areas to complex scenes in urban areas, from low-resolution images to high-resolution images.

Most methods of road extraction try to fulfil three type of tasks - detection and extraction of road segments, connection of the segments and road network construction. The process of road segments connection and building of road network takes into account the geometric characteristics of the road and the detected road cross-structure constraints to ensure the correctness of the road extraction connection results. Hand crafted feature based methods include template matching $(\mathrm{Hu}$ and Tao, 2005), tracing (Bonnefon et.al,2002;Kim et.al, 2004), line matching (Shi et.al, 2002), boosting based machine learning(Carlson et.al, 2010) and multilevel cues(Das et.al, 2011) etc. In recent years, deep learning based methods, such as DLinkNet (Zhou et.al, 2018) shows the power in extracting high accuracy roads with the cost of large amount of high quality labeled samples. On the premise of existing road vector semantic labels, the problem of road feature heterogeneity and diversity in high resolution remote sensing images still needs to be solved.

In urban areas, roads are connected and crossed by intersections and junctions, among them the most frequently appearing ones are "T" junctions and "+", "X", "Y" and intersections. While the road surface may be occluded by tall buildings and trees, road intersections are often uncovered or less covered, the detection and verification methods of road intersections can provide stable and reliable information for extraction of urban road network, with higher completeness and accuracy. In most cases road blocks in an urban area can be constructed by simply linking the intersections and junctions with their type and geometric information, which are directions of the road segments connected by the intersection. Based on the analysis, this paper proposes a method for automatic detection and recognition of road intersections for road extraction from an image of urban area. In section 2 the main steps are described,

\footnotetext{
* Corresponding author
} 
followed by the experimental results in section 3 and conclusion in section 4 .

\section{SEGMENTATION-SHAPE ANALYSIS FOR ROAD INTERSECTION DETECTION AND RECOGNITION}

\subsection{Main steps of the algorithm}

This paper proposes a method for automatic detection and recognition of road intersections for road extraction from an image of urban area. The main work flow is :
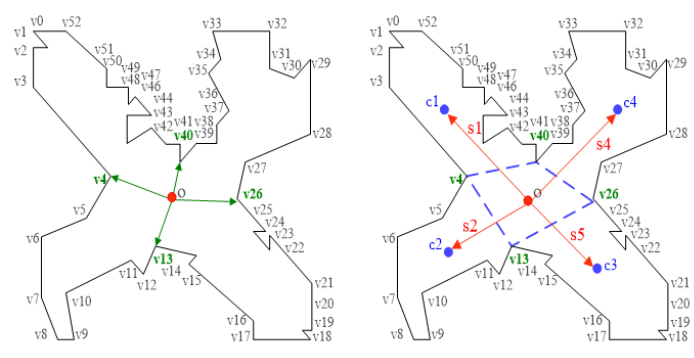

contour shape of the intersection segment road directions can be extracted

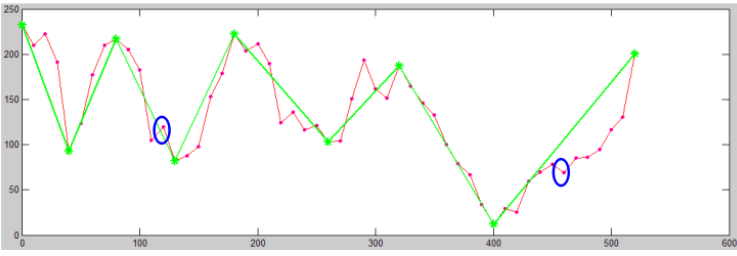

projected (red) and compressed (green) contour shape signal

Figure 1. Illustration of road intersection recognition

1. Establishing a road intersection model and extracting the initial road segments from remote sensing images;

2. Performing an intersection operation on the initial road segments to detect possible road intersections (region of interest) and constructing initial road network;

3. Detection and verification of initial road intersections based on image segmentation of the intersection area, as shown in Figure 1.

The details of the three steps are described in the following sub sections.

\subsection{Road intersection model}

As shown in Figure 2, road intersection models are typically "T" junctions and "+", "X", "Y" and intersections.
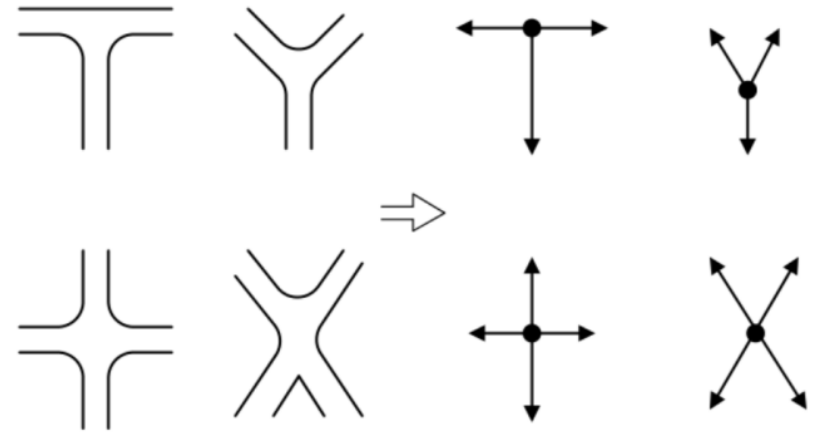

Figure 2. Road intersection models

\subsection{Intersection regions of interest}
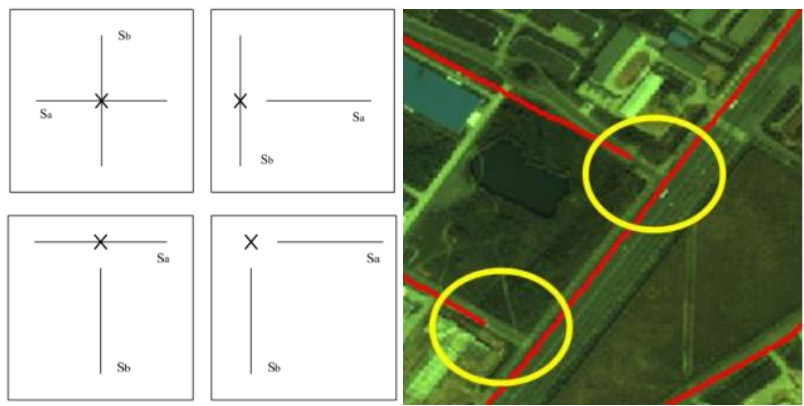

Figure 3. Intersection regions of interest

By using road detection methods, candidate road segments can be detected as $S_{a}$ and $S_{b}$ shown in figure 3. These segments intersects each other in different cases to form intersection location as " $\mathrm{X}$ " shown in the figure 3 .

\subsection{Image segmentation of the intersection regions}

After the detection of intersection locations from the last step, the regions of intersection interest can be segmented. The image segmentation is implemented by mean shift, a non-parametric iterative algorithm or a non-parametric density gradient estimation using a generalized kernel (Comaniciu and Meer, 2002). An example of the image segmentation can be found in Figure 4 . Note the road intersections marked by the arrows, they indicate the segmented regions with particular shapes which will be used for further analysis of the intersection models.
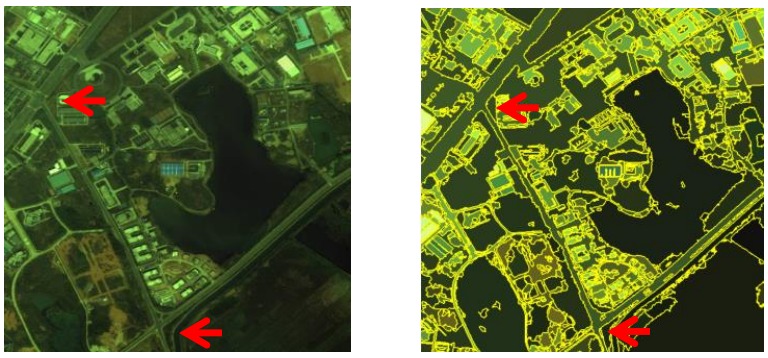

Figure 4. Image segmentation with mean shift algorithm

\subsection{Shape analysis}

It uses the contour shape of the segmented-intersection area to form a feature vector representing its geometric information. The extracted feature vector is then matched with some template vectors in order to find the best matched intersection pattern and obtain the type of intersection and the direction of connected roads; based on the type of intersection and combining its connectivity direction of the road segments, a road network of target area can be built. The algorithm works as illustrated in Figure 1:

A). Extract all pixels of contour of the intersection segment;

B). Detect feature points of the contour;

C). Compute the distance between a feature point and the center of the intersection area, and use the distances to project them into a new one-dimensional signal;

D). Use Douglas-Peucher algorithm to compress such onedimensional signal, and count the number of the wave trough of the signal; 
E). Use the wave troughs of the signal to separate different parts of the intersection segments;

F). As shown in Figure 1, in order to get the directions of each road segments connected to the intersection, a direction vector is generated by the center point of the intersection area and the center of gravity of each part, which is detected by the previous step.
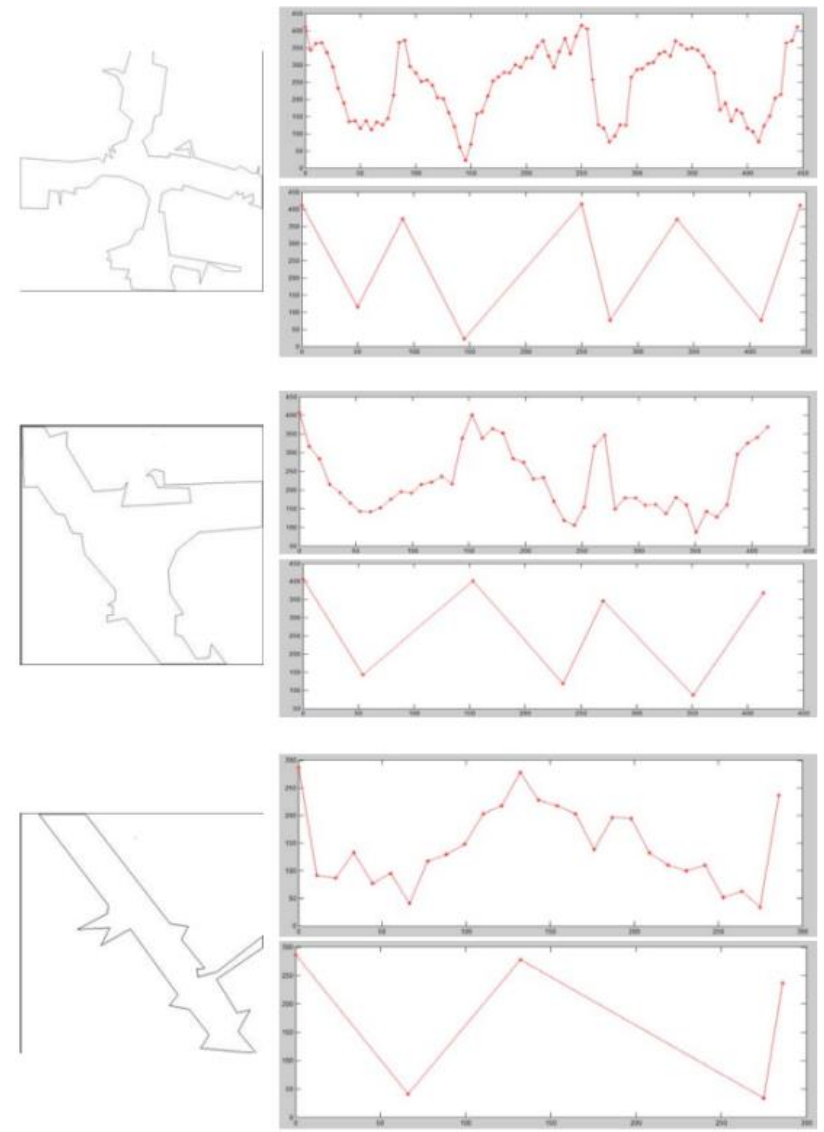

Figure 5. Examples of intersection segments and their shape representation

Figure 5 shows three typical road intersection and non intersection types. The top is a " + " intersection, the middle is a "Y" intersection, and the bottom is a road segment without intersection. After the shape representation and abstraction operation with the one dimensional signal as shown at the right the figure, we can see the road intersection pattern can be simply represented by the wave troughs of the signal and the direction vector, which is generated by the center point of the intersection area and the center of gravity of each part (see Figure 1).

\section{EXPERIMENTAL RESULTS}

The experimental results are carried out with ISPRS Vaihingen and Toronto images (ISPRS, 2010), which represent a typical European town and a North America modern city. Many parts of the roads are occluded or affected by shadows in these areas it makes ordinary road extraction very difficult. The experimental results show that the proposed method can extract most of the road intersections correctly, it can help to build more correct and complete road network. Some examples of the extraction results are shown in Figure 6.
To evaluate the quality of the result, we use the completeness and correctness to be the measurements for the two areas. The completeness and correctness are defined as

$$
\begin{aligned}
\text { correctness } & =\frac{\mathrm{TP}}{\mathrm{TP}+\mathrm{FP}} \times 100 \% \\
\text { completeness } & =\frac{\mathrm{TP}}{\mathrm{TP}+\mathrm{FN}} \times 100 \%
\end{aligned}
$$

where TP is true positive, FP is false positive, and FN is false negative. For the Vaihingen image, the the completeness and correctness are $81 \%$ and $87 \%$, respectfully, while for the Toronto image, the completeness and correctness are $78 \%$ and $85 \%$, respectfully. Note that there are many tall buildings in Toronto downtown area, even the street intersections are affected with shadows very much. Using true color images may lead to too many false results. We choose the Lidar intensity image of Toronto in the data set to do the image segmentation for the shape analysis in detection of road intersections. It proves that such intensity image can effectively overcome the shadow effect due to its reflectance property.

In figure 7, we show that in the two areas, when integrating the road intersections with the MTH (mean shift,tensor voting and Hough transform) road extraction algorithm(Hu et.al, 2014), complete and correct road networks can be extracted in such complex urban areas.
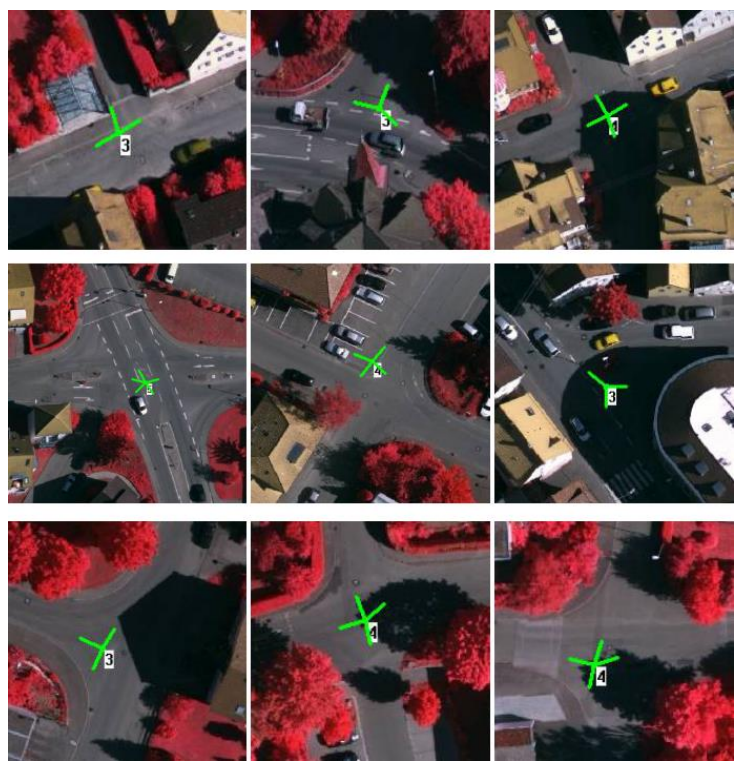

(a) Vaihingen 

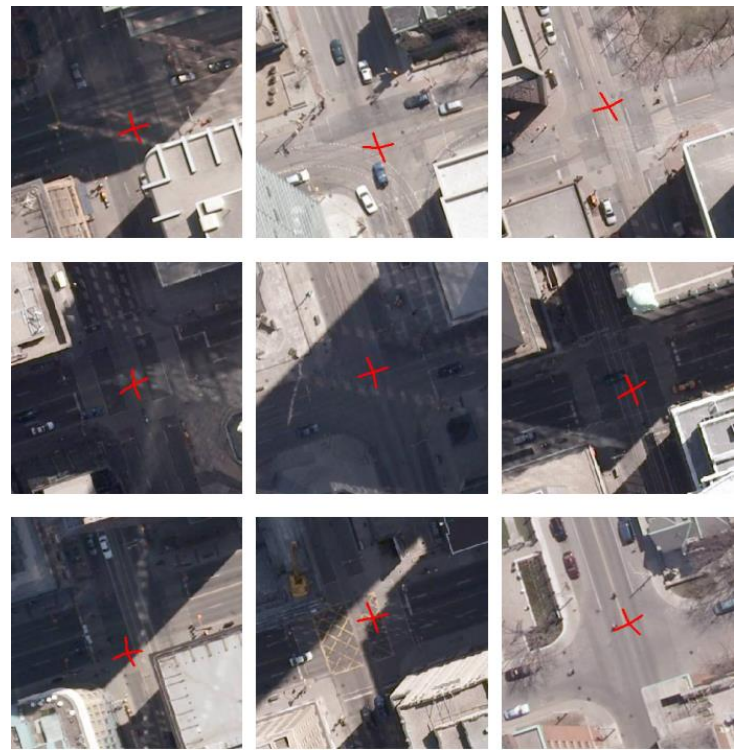

(b) Toronto

Figure 6. Results of road intersection detection and recognition

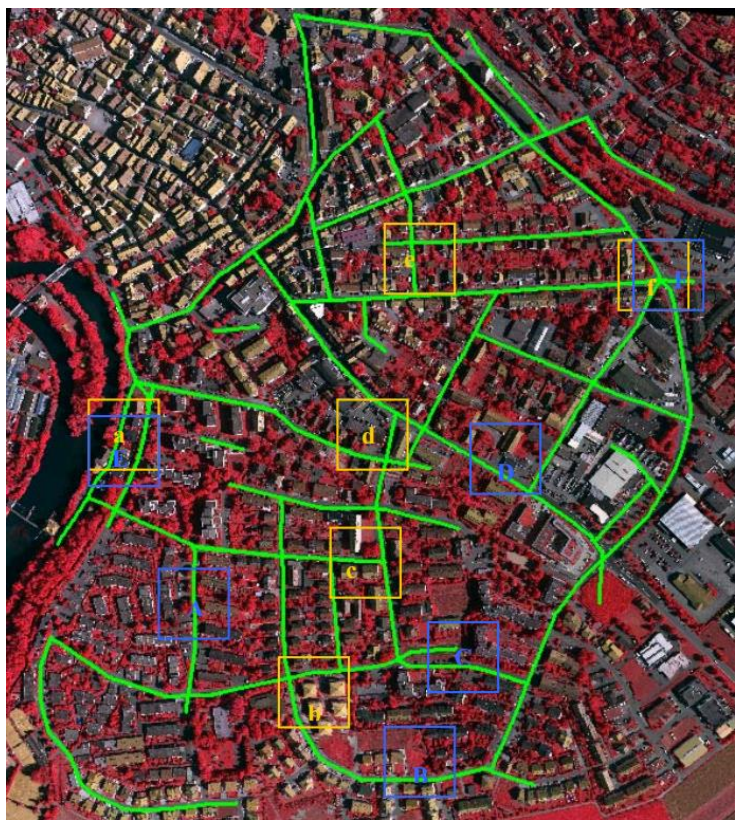

(a) Vaihingen

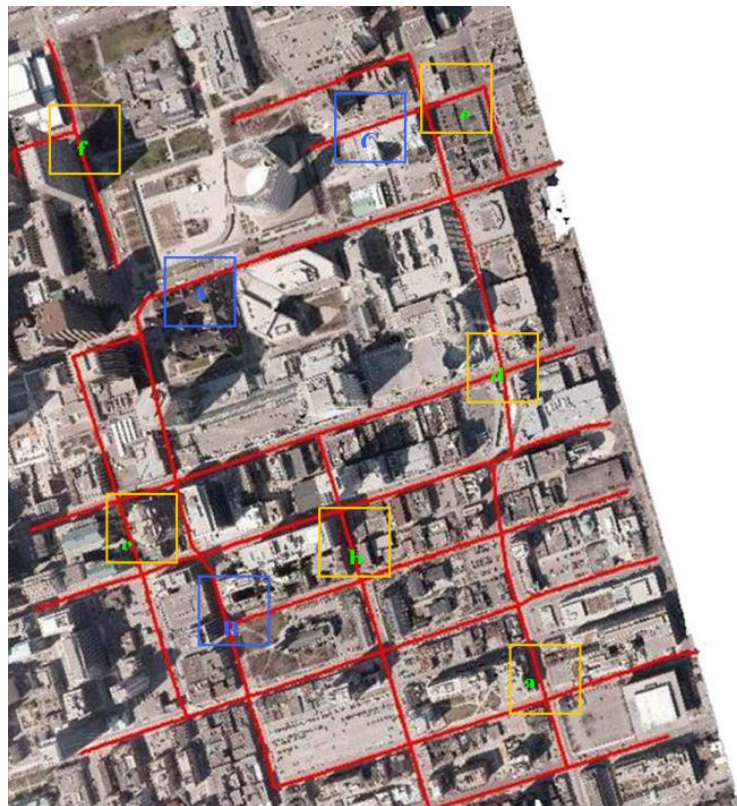

(b) Toronto

Figure 7. Road network extraction by integrating road intersections with the MTH algorithm

\section{CONCLUSION}

In this paper, a segmentation-shape analysis based method is proposed to detect road intersections and their branch directions from a remotely sensed image. The experimental results show that the proposed method can extract most of the road intersections correctly and help build a complete road network, especially in urban complex scenes. Further study includes integrating more cues to improve the correctness of the detection and using optimal algorithms to combine multiple cures.

\section{REFERENCES}

Bonnefon, R., Dherete, P., Desachy, J., 2002. Geographic information system updating using remote sensing images. Pattern Recognition Lett. 23 (9), 1073 - 1083.

Carlson. R., A. Danner., 2010. Bridge detec tion in grid terrains and improved drainage enforcement. Proceedings of the 18th SIGSPATIAL International Conference on Advances in Geographic Information Systems.

D. Comaniciu,P. Meer, 2002. A Robust Approach Toword Feanture Space Analysis. IEEE Transaction On Pattern Analysis And Maching Intelligence, 24(5):603-619.

S. Das, T. T. Mirnalinee, K. Varghese, 2011. Use of Salient Features for the Design of a Multistage Framework to Extract Roads From High-Resolution Multispectral Satellite Images. IEEE TRANSACTIONS ON GEOSCIENCE AND REMOTE SENSING, 49(10):3906-3931.

X. Hu, Y. Li, J. Shan, J. Zhang, and Y. Zhang, 2014. Road Centerline Extraction in Complex Urban Scenes From LiDAR Data Based on Multiple Features, IEEE TRANSACTIONS ON GEOSCIENCE AND REMOTE SENSING,52( 11):7448-7456. 
X. Hu, C.Tao, 2005. A Reliable and fast ribbon road detector using profile analysis and model based verification. International Journal of Remote Sensing, 26(5):887-902.

ISPRS, 2010.2D Semantic Labeling - Vaihingen datahttp://www2.isprs.org/commissions/comm3/wg4/2d-semlabel-vaihingen.html.(12 Mar 2020)

T. Kim, S. Park, M. Kim, S. Jeong, and K. Kim, 2004. Tracking Road Centerlines from High Resolution Remote Sensing Images by Least Squares Correlation Matching. Photogrammetric Engineering \& Remote Sensing, 70(12): 1417 -1422 .

Laptev, I., Mayer, H., Lindeberg, T., Eckstein, W., Steger, C.,Baumgartner, A., 2000. Automatic extraction of roads from aerial images based on scale space and snakes. Machine Vision Applicat. 12 (1), 23 - 31.

W. Shi,C. Zhu, 2002. The Line Segment Match Method for Extracting Road Network From High-Resolution Satellite Images. IEEE TRANSACTIONS ON GEOSCIENCE AND REMOTE SENSING, 40(2):511-514.

Zhou L, Zhang C, Wu M., 2018 D-linknet: Linknet with pretrained encoder and dilated convolution for high resolution satellite imagery road extraction. Proceedings of the IEEE Conference on Computer Vision and Pattern Recognition Workshops. IEEE: 182-186. 